\title{
Ocular Gene Transfer with Self-Complementary AAV Vectors
}

\author{
Katsutoshi Yokoi, ${ }^{1,2}$ Shu Kachi, ${ }^{1,2}$ H. Steve Zhang, ${ }^{3}$ Philip D. Gregory, ${ }^{3}$ S. Kaye Spratt, ${ }^{3}$ \\ R. Jude Samulski, ${ }^{4}$ and Peter A. Campochiaro ${ }^{1}$
}

PurPose. Self-complementary AAV (scAAV) vectors have been developed to circumvent rate-limiting second-strand synthesis in single-stranded AAV vector genomes and to facilitate robust transgene expression at a minimal dose. In this study, the authors investigated the effects of intraocular injections of type 2 scAAV.GFP in mice.

Methods. Dose-response experiments were performed to compare conventional single-strand AAV type 2 (ssAAV2) vectors with scAAV2 vectors encoding an identical expression cassette.

Results. Subretinal injection of $5 \times 10^{8}$ viral particles (vp) of scAAV.CMV-GFP resulted in green fluorescent protein (GFP) expression in almost all retinal pigment epithelial (RPE) cells within the area of the small detachment caused by the injection by 3 days and strong, diffuse expression by 7 days. Expression was strong in all retinal cell layers by days 14 and 28. In contrast, 3 days after subretinal injection of $5 \times 10^{8} \mathrm{vp}$ of ssAAV.CMV-GFP, GFP expression was detectable in few RPE cells. Moreover, the ssAAV vector required 14 days for the attainment of expression levels comparable to those observed using ScAAV at day 3. Expression in photoreceptors was not detectable until day 28. Dose-response experiments confirmed that onset of GFP expression was more rapid and robust after subretinal injection of scAAV.CMV-GFP than of ssAAV.CMVGFP, resulting in pronounced expression in photoreceptors and other retinal neurons. Similar results were obtained for intravitreous injections.

Conclusions. These data suggest that scAAV vectors may be advantageous for ocular gene therapy, particularly in retinal diseases that require rapid and robust transgene expression in photoreceptor cells. (Invest Ophthalmol Vis Sci. 2007;48: 3324-3328) DOI:10.1167/iovs.06-1306

A deno-associated viruses (AAVs) are nonenveloped parvoviA ruses with linear, single-stranded DNA genomes that have many characteristics that make them advantageous for use as

From the ${ }^{1}$ Departments of Ophthalmology and Neuroscience, The Johns Hopkins University School of Medicine, Baltimore, Maryland; ${ }^{3}$ Sangamo Biosciences, Richmond, California; and the ${ }^{4}$ Department of Pharmacology, University of North Carolina, Chapel Hill, North Carolina.

${ }^{2}$ Contributed equally to the work and therefore should be regarded as equivalent authors.

Supported by Sangamo Biosciences. PAC is the George S. and Dolores Dore Eccles Professor of Ophthalmology.

Submitted for publication October 31, 2006; revised January 18, 2007; accepted April 16, 2007.

Disclosure: K. Yokoi, None; S. Kachi, None; H.S. Zhang, None; P.D. Gregory, None; S.K. Spratt, None; R.J. Samulski, None; P.A. Campochiaro, Sangamo Biosciences (P)

The publication costs of this article were defrayed in part by page charge payment. This article must therefore be marked "advertisement" in accordance with 18 U.S.C. $\$ 1734$ solely to indicate this fact.

Corresponding author: Peter A. Campochiaro, Departments of Ophthalmology and Neuroscience, The Johns Hopkins University School of Medicine, Maumenee 719, 600 N. Wolfe Street, Baltimore, MD 21287-9277; pcampo@jhmi.edu. viral vectors. ${ }^{1}$ Although they readily infect a wide range of nondividing human cell types with tropism determined primarily by the capsid, which varies among serotypes, in humans they are nonpathogenic, induce a moderate immune response, and mediate long-term transgene expression. One problem with recombinant AAV vectors in certain tissue types is delayed onset of transgene expression that does not reach peak levels for up to 6 weeks. ${ }^{2}$ This can be attributed to the need for efficient synthesis of the complementary strand of the singlestranded vector genome before transgene expression. ${ }^{3}$

Self-complementary AAV (scAAV) vectors contain a doublestranded vector genome generated by deletion of the terminal resolution site (TR) from one IAAV TR, preventing the initiation of replication at the mutated end. These constructs generate single-stranded, inverted-repeat genomes, with a wildtype (wt) TR at each end and a mutated TR in the middle. ${ }^{4}$ Compared with single-strand AAV (ssAAV) vectors, scAAV vectors have been shown to improve transduction efficiency in liver, ${ }^{5,6}$ trabecular meshwork, ${ }^{7}$ and brain cells. ${ }^{8}$ In this study, we investigated the use of ScAAV vectors in the eye.

\section{Materials ANd Methods}

\section{Production of SSAAV and ScAAV Vectors}

Plasmids and vectors used in this study have been previously described. $^{4,7,8}$

\section{Intraocular Injections}

Mice were treated humanely in strict compliance with the ARVO Statement for the Use of Animals in Ophthalmic and Vision Research. Type 2 scAAV2/GFP was injected intravitreously and subretinally in one eye, and ssAAV2/GFP was injected in the other eye with a Harvard pump apparatus and pulled-glass micropipettes, as previously described. $^{9}$ Micropipettes were calibrated to deliver $1 \mu \mathrm{L}$ vehicle on depression of a foot switch. For intravitreous injections, adult female $\mathrm{BALB} / \mathrm{c}$ mice were anesthetized, and, under a dissecting microscope. The sharpened tip of a micropipette was passed through the sclera, just behind the limbus into the vitreous cavity, and the foot switch was depressed. Subretinal injections were performed with a condensing lens system on the dissecting microscope and a plastic ring filled with gonioscopic solution (Gonioscopic Prism Solution; Alcon, Fort Worth, TX), which allowed visualization of the retina during the injection. The pipette tip was passed through the sclera posterior to the limbus and was positioned just above the retina. Depression of the foot switch caused a jet of injection fluid to penetrate the retina. This technique is atraumatic, and direct visualization allows confirmation that the injection was successful because of the appearance of a small retinal detachment (bleb).

\section{Flat-Mounts and Cryosections}

Mice were humanely killed, and the eyes were removed and fixed with $4 \%$ paraformaldehyde in PBS for 1 hour and with $10 \%$ phosphatebuffered formalin overnight to make flat-mounts. The cornea and lens were removed, and the entire retina was carefully dissected from the eyecup. Radial cuts were made from the edge to the equator of the retina, and the retina was flat-mounted in aquamount with the photo- 
receptor facing down. Radial cuts were also made in eyecups and were flat-mounted with the sclera facing down (choroidal flat-mounts). For cryosections, eyes were fixed in $4 \%$ paraformaldehyde and 5\% sucrose in PBS for 1 hour and were washed with 20\% sucrose in PBS overnight. Eyes were then embedded in optimal cutting temperature embedding compound (OCT; Miles Diagnostics, Elkhart, IN). Ocular frozen sections were rinsed in PBS and mounted with aquamount. Flat-mounts and sections were examined by fluorescence microscopy (Axioskop microscope; Zeiss, Thornwood, NY), and images were digitized using a three-color charge-coupled device (CCD) video camera (IK-TU40A; Toshiba, Tokyo, Japan) and a frame grabber.

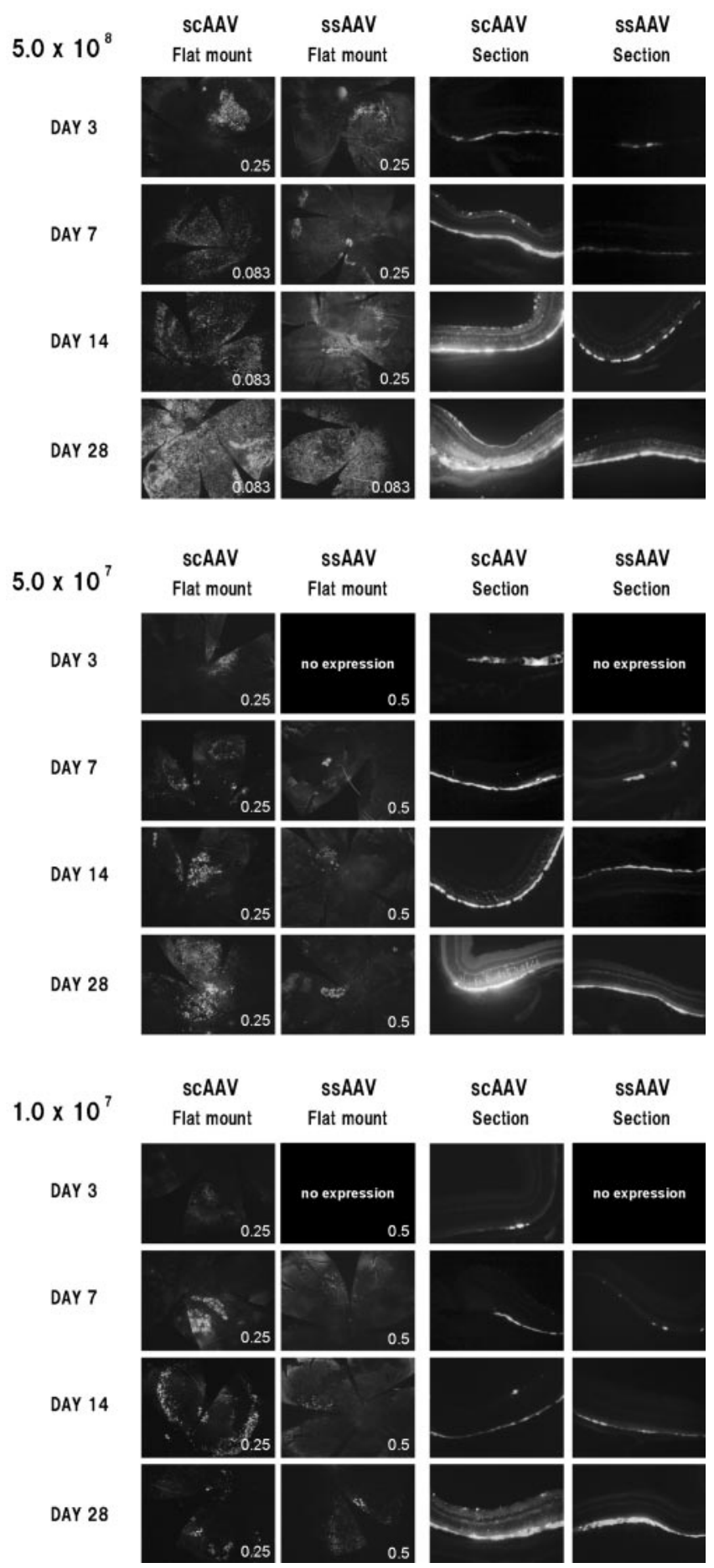

\section{Quantitative Comparison of Area of Transduced Retina}

Adult BALB/c mice underwent subretinal injection of $5 \times 10^{8}$ viral particles (vp) of scAAV.GFP in one eye and $5 \times 10^{8} \mathrm{vp}$ ssAAV.GFP in the fellow eye. At 7, 14, and 28 days after injection, five mice were humanely killed, and choroidal flat-mounts were examined by fluorescence microscopy. Images were digitized with a three-color CCD video camera and a frame grabber. Software (Image-Pro Plus; Media Cybernetics, Silver Spring, MD) was used to delineate and measure the fluorescent area per eye.

\section{Results}

\section{Subretinal Injections of Vector}

Gene transfer to RPE cells and photoreceptors requires the injection of vector into the subretinal space. Choroidal flatmounts were made 3 days after injection of $5 \times 10^{8} \mathrm{vp} \mathrm{scAAV}$. Green fluorescent protein (GFP) showed strong fluorescence throughout a moderate-sized area of RPE (Fig. 1, top panel). In fact, fluorescence intensity was saturated with 0.5 seconds of exposure; therefore, exposure was reduced to 0.25 seconds. Ocular sections showed that expression was limited to the RPE and was discontinuous. At subsequent time points, widespread strong fluorescence of the RPE was seen on choroidal flatmounts that required reduction of exposure time to 0.083 seconds. Ocular sections showed continuous transduction of RPE cells at all time points, with transduction of retinal cells ranging from focal at 7 days to strong, uniform transduction of photoreceptors and substantial transduction of cells in the inner retina at 28 days.

Three days after subretinal injection of $5 \times 10^{8} \mathrm{vp}$ ssAAV.GFP, focal areas of staining were seen on choroidal flat-mounts (Fig. 1, top panel). Fluorescence intensity was not saturated with 0.5 seconds of exposure but was still detectable at 0.25 seconds; this is shown for comparison with scAAV.GFP. Ocular sections showed occasional small areas of fluorescence in RPE cells. At subsequent time points, choroidal flat-mounts showed moderately sized areas of transduced RPE cells that were smaller and less intense than those seen with scAAV.GFP. No fluorescence was detectable after 0.083-second exposure; hence, 0.5 -second exposure images are shown. Ocular sections showed that GFP expression in retinal cells was not detectable until 28 days after injection and was less extensive and less intense than that seen after injection of scAAV.GFP.

FigURE 1. Time course of GFP reporter gene expression after subretinal injection of scAAV or ssAAV vectors (containing identical GFP expression cassettes). Adult BALB/c mice underwent subretinal injection of $5 \times 10^{8}$ (top panel), $5 \times 10^{7}$ (middle panel), or $10^{7}$ (bottom panel) vp scAAV.GFP or ssAAV.GFP. Twenty mice were injected with each dose. Results were consistent among multiple mice injected with each dose, and images shown are representative. Choroidal flat-mounts show a panoramic view of expression in RPE cells, providing an assessment of the extent (area) of transduction. Exposure time in seconds is shown in the lower-right hand corner for each image. Transduction in the overlying retina is seen in frozen sections, for which exposure was 0.5 seconds for all images. Onset of GFP expression occurred within days of scAAV.GFP or ssAAV.GFP expression, but the area and intensity of expression were greater after injection of scAAV.GFP. For both vectors, transduction of retinal cells was less efficient and expression was delayed compared with RPE cells. Expression of GFP was seen in retinal cells 7 days after injection of $5 \times 10^{8}$ vp scAAV.GFP and 28 days after injection of $5 \times 10^{8}$ vp ssAAV.GFP. With both, the extent and intensity of expression were greater after injection of scAAV.GFP. Injection of $5 \times 10^{7}$ or $10^{7} \mathrm{vp}$ resulted in uniform, strong transduction of photoreceptors for scAAV.GFP, but at these doses ssAAV.GFP failed to demonstrate GFP expression. 
The more rapid onset and the greater overall expression of GFP after subretinal injection of scAAV.GFP compared with ssAAV.GFP was confirmed by dose-dilution experiments (Fig. 1 , middle and bottom panels). Injection of $10^{7} \mathrm{vp}$ scAAV.GFP resulted in rapid onset of GFP expression in RPE cells; after 28 days, strong, uniform expression was observed in photoreceptors (Fig. 1, bottom panel). Injection of $10^{7} \mathrm{vp}$ ssAAV.GFP resulted in strong GFP expression in RPE cells by 28 days but little expression in photoreceptors. No significant fluorescence was seen after subretinal injection of $5 \times 10^{6} \mathrm{vp}$ of either vector.

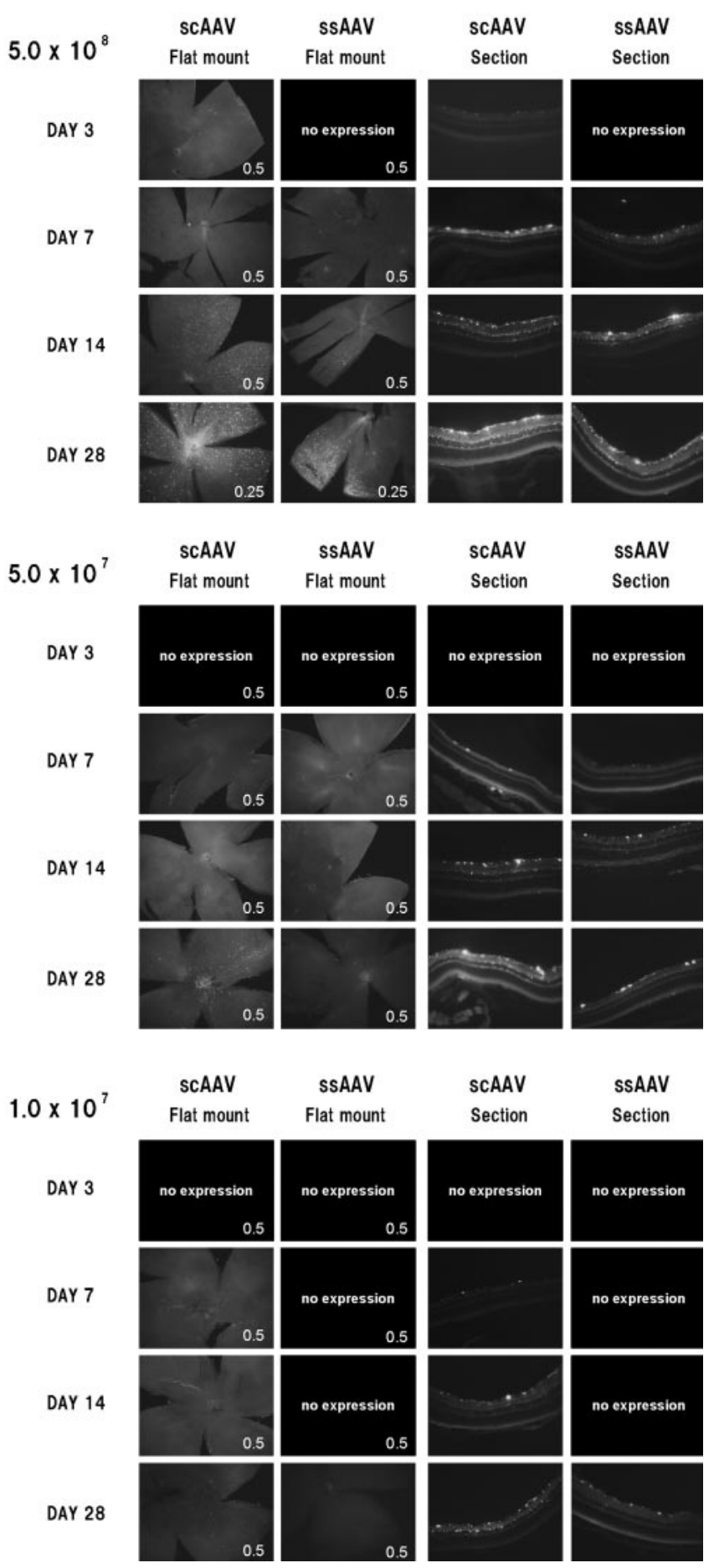

\section{Intravitreous Injection of Vector}

Onset of GFP expression occurred within 3 days of injection of $5 \times 10^{8}$ vp scAAV.GFP and within 7 days of injection of $5 \times$ $10^{8}$ vp ssAAV.GFP (Fig. 2, top panel). Although the onset of expression was later for ssAAV.GFP, by 28 days both showed transduction of ganglion cells and some cells in the inner nuclear layer throughout the entire retina. Compared with the highest dose, onset of expression was delayed after the injection of $5 \times 10^{7} \mathrm{vp} \mathrm{scAAV.GFP,} \mathrm{but} \mathrm{there} \mathrm{was} \mathrm{still} \mathrm{expression} \mathrm{of}$ GFP in ganglion cells and some cells in the inner nuclear layer throughout the retina, whereas GFP expression was limited to ganglion cells after the injection of $5 \times 10^{7} \mathrm{vp} \mathrm{ssAAV.GFP} \mathrm{(Fig.}$ 2 , middle panel). After the injection of $10^{7} \mathrm{vp}$, GFP intensity was reduced compared with that in higher doses of scAAV.GFP, but expression was still widespread in ganglion cells and some cells in the inner nuclear layer throughout the retina (Fig. 2, lower panel). In contrast, after injection of $10^{7} \mathrm{vp}$ ssAAV.GFP, no GFP expression was detectable before 28 days, and then it was limited to faint expression in ganglion cells.

\section{Quantitative Comparison of Area of Transduced RPE/Choroid}

Adult BALB/c mice underwent subretinal injection of $5 \times 10^{8}$ vp scAAV.GFP in one eye and ssAAV.GFP in the fellow eye. At 7, 14, and 28 days ( $n=5$ at each time point) after the injections, the area of fluorescence was measured in choroidal flat-mounts by image analysis. At each time point, the area of transduced RPE/choroid was significantly greater in eyes that had been injected with scAAV.GFP than in eyes injected with ssAAV.GFP (Fig. 3).

\section{Retinal Histology after Injection of scAAV Vector}

Hematoxylin-stained ocular frozen sections 7 or 14 days after subretinal injection (Figs. 4a, 4b, respectively) or intravitreous injection (Figs. $4 \mathrm{c}, 4 \mathrm{~d}$, respectively) of $5 \times 10^{8} \mathrm{vp} \mathrm{scAAV}$.GFP showed normal-appearing retinas with no evidence of inflammation.

\section{Discussion}

In this study, we have shown that compared with type 2 ssAAV vectors, type 2 scAAV vectors result in more rapid onset of transgene expression and greater transduction efficiency in various cell types in the eye. Although scAAV vectors caused modest improvement in transduction efficiency in RPE cells, the improvement in photoreceptor cells was profound, and

FIGURE 2. Time course of GFP reporter gene expression after intravitreous injection of scAAV or ssAAV vectors containing identical GFP expression cassettes. Adult BALB/c mice underwent intravitreous injection of $5 \times 10^{8}$ (top panel), $5 \times 10^{7}$ (middle panel), or $10^{7}$ (bottom panel) vp scAAV.GFP or ssAAV.GFP. Twenty mice were injected with each dose. Results were consistent among multiple mice injected with each dose, and images shown are representative. Retinal flat-mounts show a panoramic view of expression in the retina, providing an assessment of the extent (area) of transduction. Exposure time in seconds is shown in the lower right-hand corner for each image. Transduction throughout the retina was seen in frozen sections, for which exposure was 0.5 seconds for all images. Onset of GFP expression occurred within 3 days of injection of $5 \times 10^{8} \mathrm{vp} \mathrm{scAAV.GFP}$ and within 7 days of injection of $5 \times 10^{8} \mathrm{vp}$ ssAAV.GFP (top panel). Although the onset of expression was later for ssAAV.GFP than for scAAV.GFP, by 28 days both showed transduction of ganglion cells and some cells in the inner nuclear layer throughout the entire retina. Differences between scAAV.GFP and ssAAV.GFP in onset, extent, and intensity of expression were accentuated at the two lower doses of vector injected (middle and bottom panels). 


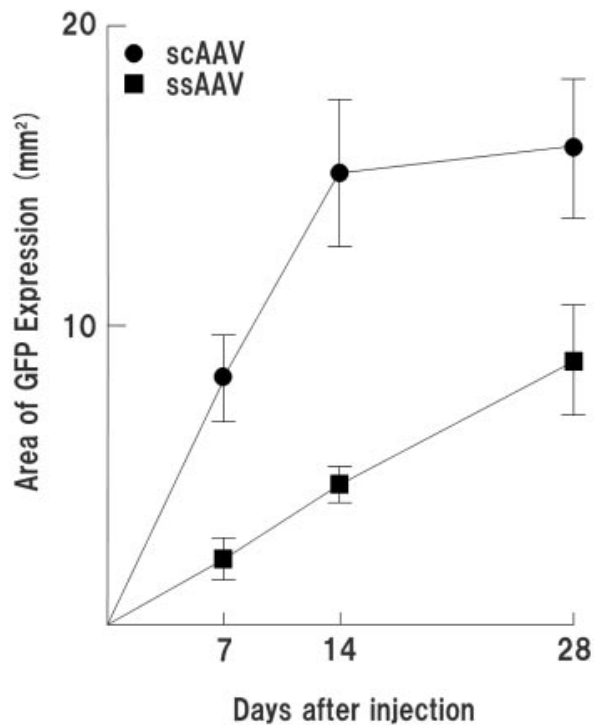

FigURE 3. Quantitative comparison of area of retina transduced by scAAV and ssAAV vectors over time after subretinal injection. Adult $\mathrm{BALB} / \mathrm{c}$ mice underwent subretinal injection of $5 \times 10^{8} \mathrm{vp} \mathrm{scAAV}$.GFP in one eye and ssAAV.GFP in the fellow eye. At 7, 14, and 28 days after the injection, five mice were humanely killed, and choroidal flatmounts were examined by fluorescence microscopy. The area of fluorescence was measured in each retina by image analysis, and each point represents the mean $( \pm$ SEM). Mean area of fluorescence per RPE/choroid was significantly greater after injection of scAAV.GFP at day $7(P=0.004183)$, day $14(0.003312)$, and day $28(0.043016)$ by Student's $t$-test.

intermediate benefit was noted in other retinal cell types. Although differences in capsid topology are likely to influence such cell type-specific tropisms, a likely explanation is that second-strand DNA synthesis is relatively efficient in RPE cells compared with ganglion cells and other cells of the inner retina and that it is inefficient in photoreceptors. This suggests that not only will scAAV vectors be advantageous for many applications of ocular gene therapy, they will be particularly useful for transgene expression in photoreceptors.

Our findings are consistent with those of previous studies showing that after subretinal injection of AAV vectors, transgene expression is detectable in RPE cells after approximately 1 week and gradually increases over several weeks, whereas photoreceptor expression is substantially more delayed and less robust. ${ }^{2,10}$ Repeated ophthalmoscopic observations after subretinal injection of ssAAV.CMV-GFP showed that fluorescence was first detectable 7 days after injection, was modest and limited in area at 13 days, and was substantially increased by 6 weeks. ${ }^{2}$ Predominant cell types expressing GFP through the 3-week time point were ganglion cells and RPE cells, with minimal transduction of photoreceptor cells. Use of a murine rhodopsin promoter to drive transgene expression in photoreceptors serves to partially recover expression from AAV-mediated gene transfer to photoreceptors but would not be expected to improve the kinetics of expression. ${ }^{11}$ Indeed, all test animals given subretinal injections of AAV.mOpsin.GFP showed strong reporter gene expression in photoreceptors 6 to 8 weeks after injection, whereas at 3 weeks expression was generally weak or below the limits of detection. The speed of onset of expression from AAV vectors in photoreceptors can be increased somewhat by $\gamma$-irradiation or other mediators of cellular stress, ${ }^{10}$ probably through the activation of DNA repair pathways that accelerate second-strand synthesis. The use of scAAV vectors offers a safer and more efficient alternative to the interventions described here.

Intravitreous injections of AAV vectors can be used to enhance or reduce intracellular proteins in ganglion cells or to produce secreted proteins in ganglion cells that can have remote effects throughout the retina. However, the enhanced transduction efficiency of scAAV vectors demonstrates that penetration of AAV vectors into the retinal tissue appears to be greater than previously thought. AAV vectors are small; hence, it is not surprising that they penetrate the retina, but internal cell layers are exposed to lower vector concentrations than cells at the surface. Concentrations achieved around cells within internal layers of the retina appear to be near the limit of detectable transduction with all but the highest intravitreous dose $\left(5 \times 10^{8}\right)$ of ssAAV vector, but good transduction of deep layers is seen with all doses of scAAV vectors. The ability to penetrate the retina after subretinal injection might have been less than that after intravitreous injection because, after subretinal injection of the highest dose, transduction of internal cells was seen only with scAAV and not with ssAAV. The greater transduction of cells within the retina after intravitreous injection provides another potential advantage for scAAV vectors for applications in which retinal cells are used as factories to produce therapeutic proteins, such as secreted angiostatic proteins in patients with ocular neovascularization or secreted neurotrophic factors in patients with retinal degen-
FIGURE 4. Retinal histology after injection of scAAV vector. Adult BALB/C mice underwent subretinal $(\mathbf{a}, \mathbf{b})$ or intravitreous (c, d) injection of $1 \mu \mathrm{L}$ of $5 \times 10^{8}$ scAAV.GFP and were humanely killed $7(\mathbf{a}, \mathbf{c})$ or $14(\mathbf{b}, \mathbf{d})$ days after injection. Ocular frozen sections stained with hematoxylin show normal retinal histology with no evidence of inflammation. Bars indicate $100 \mu \mathrm{m}$.
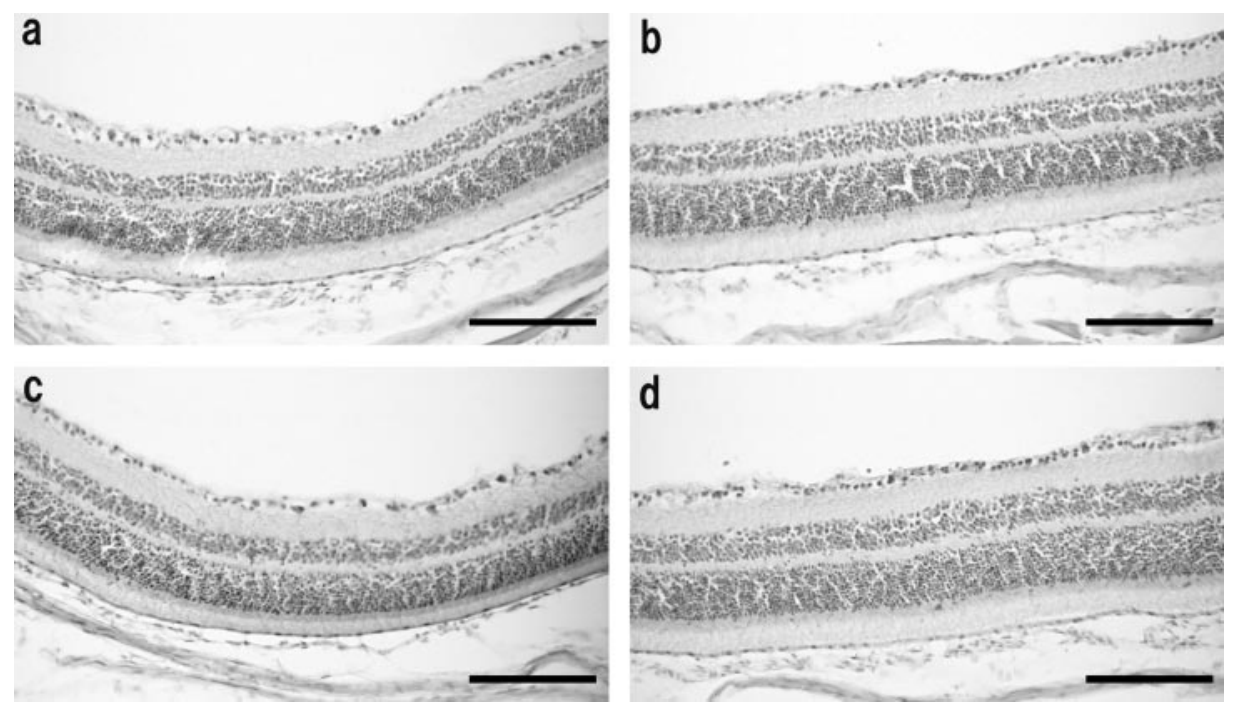
erations. Intravitreous injections are appealing because they can be performed in an outpatient setting, and, if a sufficient number of cells are transduced, a single injection may have a therapeutic effect for months or even years. A single subretinal injection of type $2 \mathrm{AAV}$. $\beta$-actin promoter.RPE65 in dogs with Leber congenital amaurosis resulted in recovery of electrical activity in the retina for at least 3 years. ${ }^{12}$ In addition, the use of scAAV vectors can facilitate studies aimed at identifying efficacious transgenes because several models occur in neonatal animals requiring rapid onset of expression after vector injection so that technically difficult and less precise intraocular injections can be avoided in utero or in newborns. Ocular neovascularization occurs within 2 weeks of birth in mice with oxygen-induced ischemic retinopathy ${ }^{13}$ and rho/VEGF transgenic mice. ${ }^{14}$ Retinal degeneration begins around postnatal day 10 in $r d 1$ mice. $^{15}$

Gene transfer to RPE cells or photoreceptors requires subretinal injection, which is a more involved but clearly feasible surgical procedure. The use of scAAV may be particularly valuable for gene transfer to photoreceptors, wherein the difference between gene expression achieved using scAAV or ssAAV was profound. A disadvantage of scAAV vectors is the more severe size limitation for expression cassettes; the cDNA for transgenes and associated regulatory elements cannot exceed $2.5 \mathrm{~kb}$, which is approximately half the allowable length for ssAAV vectors. Therefore, it is critical to understand all parameters of the application to decide between ScAAV and ssAAV, including target cell type, desired transgene expression levels, size of the expression construct, and the importance of rapid onset of expression and minimization of vector dose. Although ssAAV vectors may be perfectly adequate or even preferable for certain applications, the availability of scAAV vectors clearly expands the potential applications of AAVmediated ocular gene therapy.

\section{References}

1. Grimm D, Kay MA. From virus evolution to vector revolution: use of naturally occurring serotypes of adeno-associated virus (AAV) as novel vectors for human gene therapy. Curr Gene Ther. 2003;3: 281-304.

2. Bennett J, Duan D, Engelhardt JF, Maguire AM. Real-time, noninvasive in vivo assessment of adeno-associated virus-mediated retinal transduction. Invest Ophthalmol Vis Sci. 1997;38:2857-2863.
3. Ferrari FK, Samulski T, Shenk T, Samulski RJ. Second-strand synthesis is a rate limiting step for efficient transduction by recombinant adeno-associated virus vectors. J Virol. 1996;70:27-34.

4. McCarty DM, Monahan PE, Samulski RJ. Self-complementary recombinant adeno-associated virus (scAAV) vectors promote efficient transduction independently of DNA synthesis. Gene Ther. 2001;8:1248-1254.

5. Nathwani AC, Gray JT, Ng CY, et al. Self-complementary adenoassociated virus vectors containing a novel liver-specific human factor IX expression cassette enable highly efficient transduction of murine and nonhuman primate liver. Blood. 2006;107:26532661.

6. Gao G-P, Lu Y, Sun X, et al. High-level transgene expression in nonhuman primate liver with novel adeno-associated virus serotypes containing self-complementary genomes. J Virol. 2006;80: 6192-6194.

7. Borras T, Xue W, Choi VW, et al. Mechanisms of AAV transduction in glaucoma-associated human trabecular meshwork cells. J Gene Med. 2006;8:589-602.

8. Fu H, Muenzer J, Samulski RJ, et al. Self-complementary adenoassociated virus serotype 2 vector: global distribution and broad dispersion of AAV-mediated transgene expression in mouse brain. Mol Ther. 2004;8:911-917.

9. Mori K, Ando A, Gehlbach P, et al. Inhibition of choroidal neovascularization by intravenous injection of adenoviral vectors expressing secretable endostatin. Am J Pathol. 2001;159:313-320.

10. Ali RR, Reichel MB, De Alwis M, et al. Adeno-associated virus gene transfer to mouse retina. Hum Gene Ther. 1998;9:81-86.

11. Flannery JG, Zolotukhin S, Vaquero MI, LaVail MM, Muzyczka N, Hauswirth WW. Efficient photoreceptor-targeted gene expression in vivo by recominant adeno-associated virus. Proc Natl Acad Sci USA. 1997;94:6916-6921.

12. Acland GM, Aguirre GD, Bennett J, et al. Long-term restoration of rod and cone vision by single dose rAAV-mediated gene transfer to the retina in a canine model of childhood blindness. Mol Ther. 2005; 12:1072-1082.

13. Smith LEH, Wesolowski E, McLellan A, et al. Oxygen-induced retinopathy in the mouse. Invest Ophthalmol Vis Sci. 1994;35: 101-111.

14. Okamoto N, Tobe $\mathrm{T}$, Hackett SF, et al. Transgenic mice with increased expression of vascular endothelial growth factor in the retina: a new model of intraretinal and subretinal neovascularization. Am J Pathol. 1997;151:281-291.

15. Carter-Dawson LD, LaVail MM, Sidman RL. Differential effects of the $r d$ mutation on rods and cones in the mouse retina. Invest Ophthalmol Vis Sci. 1978;17:489-498. 